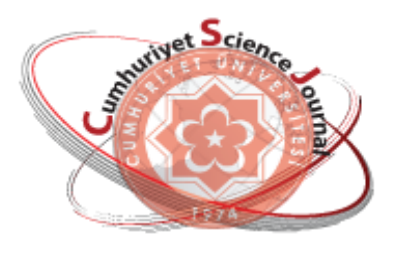

e-ISSN: 2587-246X

ISSN: 2587-2680

\section{Cumburiyet Scionce Journal \\ esy}

Cumhuriyet Sci. J., Vol.38-4, Supplement (2017) 1-5

\title{
Some New Integral Inequalities for n-Times Differentiable Godunova-Levin Functions
}

\author{
Huriye KADAKAL ${ }^{1}$, Mahir KADAKAL ${ }^{2 *}$, Imdat ISCAN ${ }^{2}$ \\ ${ }^{1}$ Institute of Science, Ordu University-Ordu /TÜRKIYE \\ ${ }^{2}$ Department of Mathematics, Faculty of Sciences and Arts, Giresun University-Giresun / TÜRKIYYE
}

http://dx.doi.org/10.17776/csj.358766

\begin{abstract}
In this work, by using an integral identity together with the Hölder integral inequality we establish several new inequalities for $n$-times differentiable Godunova-Levin functions

Keywords: Convex function, Godunova-Levin function, Hölder Integral inequality.

\section{n-kere Türevlenebilen Godunova-Levin Fonksiyonları için Bazı Yeni İntegral Eşitsizlikler}

Özet: Bu çalışmada, Hölder integral eşitsizliği ile birlikte bir integral eşitliği kullanılarak n-kere türevlenebilen Godunova-Levin Fonksiyonları için bir kaç yeni eşitsizlik bulunmuştur.

Anahtar Kelimeler: Konveks fonksiyon, Godunova-Levin fonksiyonu, Hölder İntegral eşitsizliği.

\section{INTRODUCTION}

Theory of convex functions plays an important role in different fields of pure and applied sciences. Recently much attention has been given to theory of convex functions by many researchers. In this paper, by using the Hölder integral inequality, we establish some new inequalities for functions whose $n$th derivatives in absolute value are convex functions. For some inequalities, generalizations and applications concerning convexity see [5-12,
21]. Recently, in the literature there are so many papers about $n$-times differentiable functions on several kinds of convexities. In references [2-4, 11, $14,17,19,20]$, readers can find some results about this issue. Many papers have been written by a number of mathematicians concerning inequalities for different classes of convex and GodunovaLevin functions see for instance the recent papers $[1,13,15,16,18]$ and the references within these papers.

Definition 1.1: A function $f: I \subseteq \mathbb{R} \rightarrow \mathbb{R}$ is said to be convex if the inequality

$$
f(t x+(1-t) y) \leq t f(x)+(1-t) f(y)
$$

is valid for all $x, y \in I$ and $t \in[0,1]$. If this inequality reverses, then $f$ is said to be concave on interval $I \neq \emptyset$. This definition is well known in the literature. 
Definition 1.2: A function $f: I \subseteq \mathbb{R} \rightarrow \mathbb{R}$ is said to be Godunova-Levin function, if

where $\forall x, y \in I, t \in(0,1)$.

$$
f(t x+(1-t) y) \leq \frac{f(x)}{t}+\frac{f(y)}{1-t}
$$

Throughout this paper we will use the following notations and conventions. Let $\quad J=[0, \infty) \subset$ $\mathbb{R}=(-\infty,+\infty)$, and $a, b \in J$ with $0<a<b$ and

$$
A(a, b)=\frac{a+b}{2}, \quad L_{p}(a, b)=\left(\frac{b^{p+1}-a^{p+1}}{(p+1)(b-a)}\right)^{\frac{1}{p}}, a \neq b, p \in \mathbb{R}, \quad p \neq-1,0
$$

be the arithmetic, geometric, identic, harmonic, logarithmic, generalized logarithmic mean for $a, b>0$ respectively.

For we obtain the main results we will use the following Lemma [14].

Lemma 1.1: Let $f: I \subseteq \mathbb{R} \rightarrow \mathbb{R}$ be $n$-times differentiable mapping on $I^{\circ}$ for $n \in \mathbb{N}$ and $f^{(n)} \in L[a, b]$, where $a, b \in I^{\circ}$ with $a<b$, we have the identity

$$
\sum_{k=0}^{n-1}(-1)^{k}\left(\frac{f^{(k)}(b) b^{k+1}-f^{(k)}(a) a^{k+1}}{(k+1) !}\right)-\int_{a}^{b} f(x) d x=\frac{(-1)^{n+1}}{n !} \int_{a}^{b} x^{n} f^{(n)}(x) d x .
$$

\section{MAIN RESULTS}

Theorem 2.1. For $\forall n \in \mathbb{N}$; let $f: I \subset(0, \infty) \rightarrow \mathbb{R}$ be $n$-times differentiable function on $I^{\circ}$ and $a, b \in I^{\circ}$ with $a<b$. If $\left|f^{(n)}\right|^{q}$ for $q>1$ is Godunova-Levin function on $[a, b]$, then the following inequality holds:

$$
\begin{aligned}
& \left|\sum_{k=0}^{n-1}(-1)^{k}\left(\frac{f^{(k)}(b) b^{k+1}-f^{(k)}(a) a^{k+1}}{(k+1) !}\right)-\int_{a}^{b} f(x) d x\right| \\
& \leq \frac{1}{n !}(b-a)^{\frac{3}{q}} C^{\frac{1}{p}}(a, b, n, p) A^{\frac{1}{q}}\left(\left|f^{(n)}(a)\right|^{q},\left|f^{(n)}(b)\right|^{q}\right)
\end{aligned}
$$

Where $\frac{1}{p}+\frac{1}{q}=1,1<p<2$ and $C(a, b, n, p)=\int_{a}^{b} \frac{x^{n p}}{(x-a)^{p-1}(b-x)^{p-1}} d x$.

Proof. If $\left|f^{(n)}\right|^{q}$ for $q>1$ is Godunova-Levin function on $[a, b]$, using Lemma1.1, the Hölder integral inequality and

$$
\begin{gathered}
\left|f^{(n)}(x)\right|^{q}=\left|f^{(n)}\left(\frac{x-a}{b-a} b+\frac{b-x}{b-a} a\right)\right|^{q} \leq \frac{\left|f^{(n)}(b)\right|^{q}}{\frac{x-a}{b-a}}+\frac{\left|f^{(n)}(a)\right|^{q}}{\frac{b-x}{b-a}}, \\
\left|f^{(n)}(x)\right|^{q} \leq \frac{b-a}{x-a}\left|f^{(n)}(b)\right|^{q}+\frac{b-a}{b-x}\left|f^{(n)}(a)\right|^{q} \\
(x-a)(b-x)\left|f^{(n)}(x)\right|^{q} \leq(b-a)(b-x)\left|f^{(n)}(b)\right|^{q}+(b-a)(x-a)\left|f^{(n)}(a)\right|^{q}
\end{gathered}
$$

we have

$\left|\sum_{k=0}^{n-1}(-1)^{k}\left(\frac{f^{(k)}(b) b^{k+1}-f^{(k)}(a) a^{k+1}}{(k+1) !}\right)-\int_{a}^{b} f(x) d x\right| \leq \frac{1}{n !} \int_{a}^{b} x^{n}\left|f^{(n)}(x)\right| d x$ 


$$
\begin{aligned}
& \leq \frac{1}{n !}\left(\int_{a}^{b} \frac{x^{n p}}{(x-a)^{p-1}(b-x)^{p-1}} d x\right)^{\frac{1}{p}}\left(\int_{a}^{b}(x-a)(b-x)\left|f^{(n)}(x)\right|^{q} d x\right)^{\frac{1}{q}} \\
& \leq \frac{1}{n !} C^{\frac{1}{p}}(a, b, n, p)\left(\int_{a}^{b}\left\{(b-a)(b-x)\left|f^{(n)}(b)\right|^{q}+(b-a)(x-a)\left|f^{(n)}(a)\right|^{q}\right\} d x\right)^{\frac{1}{q}} \\
& =\frac{1}{n !} C^{\frac{1}{p}}(a, b, n, p)\left(\left.(b-a)\left|f^{(n)}(b)\right|^{q}\left(b x-\frac{x^{2}}{2}\right)\right|_{a} ^{b}+\left.(b-a)\left|f^{(n)}(a)\right|^{q}\left(\frac{x^{2}}{2}-a x\right)\right|_{a} ^{b}\right)^{\frac{1}{q}} \\
& =\frac{1}{n !} C^{\frac{1}{p}}(a, b, n, p)\left[(b-a)\left|f^{(n)}(b)\right|^{q} \frac{(b-a)^{2}}{2}+(b-a)\left|f^{(n)}(a)\right|^{q} \frac{(b-a)^{2}}{2}\right]^{\frac{1}{q}} \\
& =\frac{1}{n !} C^{\frac{1}{p}}(a, b, n, p)\left[\left|f^{(n)}(b)\right|^{q} \frac{(b-a)^{3}}{2}+\left|f^{(n)}(a)\right|^{q} \frac{(b-a)^{3}}{2}\right]^{\frac{1}{q}} \\
& =\frac{1}{n !} C^{\frac{1}{p}}(a, b, n, p)(b-a)^{\frac{3}{q}}\left[\frac{\left|f^{(n)}(b)\right|^{q}+\left|f^{(n)}(a)\right|^{q}}{2}\right]^{\frac{1}{q}} \\
& =\frac{1}{n !} C^{\frac{1}{p}}(a, b, n, p)(b-a)^{\frac{3}{q}} A^{\frac{1}{q}}\left(\left|f^{(n)}(a)\right|^{q},\left|f^{(n)}(b)\right|^{q}\right)
\end{aligned}
$$

It is stated that the improper integral $C(a, b, n, p)$ is convergent for $1<p<2$.

Corollary 2.1. Under the conditions Theorem 2.1 for $n=1$ we have the following inequality:

$$
\left|\frac{f(b) b-f(a) a}{b-a}-\frac{1}{b-a} \int_{a}^{b} f(x) d x\right| \leq C^{\frac{1}{p}}(a, b, 1, p)(b-a)^{\frac{3}{q}-1} A^{\frac{1}{q}}\left(\left|f^{\prime}(a)\right|^{q},\left|f^{\prime}(b)\right|^{q}\right) .
$$

Theorem 2.2. For $n \in \mathbb{N}$; let $f:(0, \infty) \subset \mathbb{R} \rightarrow \mathbb{R}$ be $n$-times differentiable function and $\quad 0 \leq a<b$. If $\left|f^{(n)}\right|^{q} \in L[a, b]$ and $\left|f^{(n)}\right|^{q}$ for $q>1$ is Godunova-Levin function on $[a, b]$, then the following inequality holds:

$$
\begin{aligned}
& \left|\sum_{k=0}^{n-1}(-1)^{k}\left(\frac{f^{(k)}(b) b^{k+1}-f^{(k)}(a) a^{k+1}}{(k+1) !}\right)-\int_{a}^{b} f(x) d x\right| \leq \frac{1}{n !}(b-a)^{\frac{2}{q}} D^{\frac{1}{p}}(a, b, n, p) \\
& \times\left[\left|f^{(n)}(b)\right|^{q}\left\{b L_{n}^{n}(a, b)-L_{n+1}^{n+1}(a, b)\right\}+\left|f^{(n)}(a)\right|^{q}\left\{L_{n+1}^{n+1}(a, b)-a L_{n}^{n}(a, b)\right\}\right]^{\frac{1}{q}}
\end{aligned}
$$

Where $\frac{1}{p}+\frac{1}{q}=1,1<p<2$ and $D(a, b, n, p)=\int_{a}^{b} \frac{x^{n}}{(x-a)^{p-1}(b-x)^{p-1}} d x$.

Proof. From Lemma1.1 and Hölder integral inequality, we obtain

$$
\begin{aligned}
& \left|\sum_{k=0}^{n-1}(-1)^{k}\left(\frac{f^{(k)}(b) b^{k+1}-f^{(k)}(a) a^{k+1}}{(k+1) !}\right)-\int_{a}^{b} f(x) d x\right| \leq \frac{1}{n !} \int_{a}^{b} x^{n}\left|f^{(n)}(x)\right| d x \\
& \leq \frac{1}{n !}\left(\int_{a}^{b} \frac{x^{n}}{(x-a)^{p-1}(b-x)^{p-1}} d x\right)^{\frac{1}{p}}\left(\int_{a}^{b} x^{n}(b-x)(x-a)\left|f^{(n)}(x)\right|^{q} d x\right)^{\frac{1}{q}}
\end{aligned}
$$




$$
\begin{aligned}
& \leq \frac{1}{n !} D^{\frac{1}{p}}(a, b, n, p)\left[\int_{a}^{b} x^{n}\left[(b-a)(b-x)\left|f^{(n)}(b)\right|^{q}+(b-a)(x-a)\left|f^{(n)}(a)\right|^{q}\right] d x\right]^{\frac{1}{q}} \\
& \leq \frac{1}{n !} D^{\frac{1}{p}}(a, b, n, p) \\
& \times\left[(b-a)\left|f^{(n)}(b)\right|^{q}\left\{b\left(\frac{b^{n+1}-a^{n+1}}{n+1}\right)-\left(\frac{b^{n+2}-a^{n+2}}{n+2}\right)\right\}\right. \\
& \left.\quad+(b-a)\left|f^{(n)}(a)\right|^{q}\left\{\left(\frac{b^{n+2}-a^{n+2}}{n+2}\right)-a\left(\frac{b^{n+1}-a^{n+1}}{n+1}\right)\right\}\right]^{\frac{1}{q}} \\
& \leq \frac{1}{n !} D^{\frac{1}{p}}(a, b, n, p) \\
& \times\left[(b-a)^{2}\left|f^{(n)}(b)\right|^{q}\left\{b\left(\frac{b^{n+1}-a^{n+1}}{(b-a)(n+1)}\right)-\left(\frac{b^{n+2}-a^{n+2}}{(b-a)(n+2)}\right)\right\}\right. \\
& \left.\quad+(b-a)^{2}\left|f^{(n)}(a)\right|^{q}\left\{\left(\frac{b^{n+2}-a^{n+2}}{(b-a)(n+2)}\right)-a\left(\frac{b^{n+1}-a^{n+1}}{(b-a)(n+1)}\right)\right\}\right]^{\frac{1}{q}} \\
& \leq \frac{1}{n !}(b-a)^{\frac{2}{q}} D^{\frac{1}{p}(a, b, n, p)} \\
& \times\left[\left|f^{(n)}(b)\right|^{q}\left\{b L_{n}^{n}(a, b)-L_{n+1}^{n+1}(a, b)\right\}+\left|f^{(n)}(a)\right|^{q}\left\{L_{n+1}^{n+1}(a, b)-a L_{n}^{n}(a, b)\right\}\right]^{\frac{1}{q}}
\end{aligned}
$$

It is stated that the improper integral $D(a, b, n, p)$ is convergent for $1<p<2$.

Corollary 2.2. Under the conditions Theorem 2.2 for $n=1$ we have the following inequality:

$$
\begin{aligned}
& \left|\frac{f(b) b-f(a) a}{b-a}-\frac{1}{b-a} \int_{a}^{b} f(x) d x\right| \\
& \leq\left(\frac{1}{6}\right)^{\frac{1}{q}}(b-a)^{\frac{3}{q}-1} D^{\frac{1}{p}}(a, b, 1, p)\left[(b+2 a)\left|f^{\prime}(b)\right|^{q}+(2 b+a)\left|f^{\prime}(a)\right|^{q}\right]^{\frac{1}{q}} .
\end{aligned}
$$

\section{REFERENCES}

[1]. Akdemir A.O., Özdemir M. E., "Some Hadamard-Type Inequalities For Coordinated P-Convex Functions and Godunova-Levin Functions", arXiv:1012.5880v2 [math.CA] 24 Mar 2011.

[2]. Cerone P., Dragomir S.S., Roumeliotis J., "Some Ostrowski type inequalities for n-time differentiable mappings and applications", Demonstratio Math., 32 (4) (1999), 697-712.

[3]. Cerone P., Dragomir S.S., Roumeliotis J., Šunde J., "A new generalization of the trapezoid formula for n-time differentiable mappings and applications", Demonstratio Math., 33 (4) (2000), 719-736.
[4]. Hwang D.Y.,"Some Inequalities for n-time Differentiable Mappings and Applications", Kyung. Math. Jour., 43 (2003), 335-343.

[5]. İşcan İ., "Some Generalized HermiteHadamard Type Inequalities for QuasiGeometrically Convex Functions", American Journal of Mathematical Analysis, 2013, Vol. 1, No. 3, 48-52.

[6]. İşcan İ., "Some new general integral inequalities for $h$-convex and $h$-concave functions", Adv. Pure Appl. Math. 5 (1), 21 29 (2014).

[7]. İşcan İ., "Hermite-Hadamard type inequalities for harmonically convex functions", 
Hacettepe Journal of Mathematics and Statistics, Volume 43 (6) (2014), 935-942.

[8]. İşcan İ., "Hermite-Hadamard type inequalities for GA-s-convex functions", Le Matematiche, Vol. LXIX (2014) Fasc. II, pp. 129-146

[9]. İşcan İ., Numan S., "Ostrowski type inequalities for harmonically quasi-convex functions", Electronic Journal of Mathematical Analysis and Applications, Vol. 2(2) July 2014, pp. 189-198.

[10]. İşcan İ., "On new general integral inequalities for quasi-convex functions and their applications", Palestine Journal of Mathematics. 4(1) (2015), 21-29.

[11]. Jiang W.D., Niu D.W., Hua Y., Qi F., "Generalizations of Hermite-Hadamard inequality to n-time differentiable function which are s -convex in the second sense", Analysis (Munich), 32 (2012), 209-220.

[12]. Kırmacı U.S., Bakula M.K., Özdemir M.E., Pećarić J., "Hadamard-type inequalities for s convex functions", Appl. Math. and Comp., 193 (2007), 26-35.

[13]. Li M., Andweiwei J., "Some Fractional Hermite-Hadamard Inequalities for Convex and Godunova-Levin Functions", Ser. Math. Inform. Vol. 30, No 2 (2015), 195-208.

[14]. Maden S., Kadakal H., Kadakal M., İşcan İ., "Some new integral inequalities for n-times differentiable convex and concave functions". https://www.researchgate.net/publication/312 529563, (Submitted).

[15]. Noor M.A., Noor K.I., Awan M.U., "Fractional Ostrowski Inequalities for $\mathrm{s}$ Godunova-Levin Functions", International Journal of Analysis and Applications, Volume 5, Number 2 (2014), 167-173.

[16]. Noor M.A., Noor K.I., Awan M.U., Khan S., "Fractional Hermite-Hadamard Inequalities for some New Classes of Godunova-Levin Functions", Appl. Math. Inf. Sci. 8, No. 6, 2865-2872, (2014).

[17]. Özdemir M.E., Yıldız Ç., "New Inequalities for n-time differentiable functions", Arxiv:1402.4959v1.

[18]. Özdemir M.E., "Some inequalities for the sGodunova-Levin type functions", Math Sci (2015) 9:27-32.

[19]. Set E., Özdemir M.E., Dragomir S.S., "On Hadamard-Type Inequalities Involving Several Kinds of Convexity", Jour. of Ineq. and Appl., 2010, 286845.

[20]. Wang S.H., Xi B.Y., Qi F., "Some new inequalities of Hermite-Hadamard type for $\mathrm{n}$ time differentiable functions which are $\mathrm{m}$ convex", Analysis (Munich), 32 (2012), 247262.

[21]. Xi B. Y., Qi F., "Some Integral Inequalities of Hermite-Hadamard Type for Convex Functions with Applications to Means", J. Funct. Spaces Appl (2012) 14 pages 